\title{
Immediate effects of Zhongji point acupuncture on pelvic floor structure in female patients with stress urinary incontinence: a randomized, single-blind, and sham-controlled clinical trial protocol
}

\author{
Li-Xin Guan $^{1 \#}$, Xiu-Ling Song ${ }^{2 *} \wedge$, Xi Wang ${ }^{3}$, Xia Zhang $^{3}$, Lu-Min Liu ${ }^{1}$, Bing-Li Chen ${ }^{1}$, Yue-Lai Chen ${ }^{1 \wedge}$ \\ ${ }^{1}$ Yueyang Hospital of Integrated Traditional Chinese and Western Medicine, Shanghai University of Traditional Chinese Medicine, Shanghai, China; \\ ${ }^{2}$ Shanghai University of Traditional Chinese Medicine, Shanghai, China; ${ }^{3}$ Xuhui Hospital, Zhongshan Hospital, Fudan University, Shanghai, China \\ Contributions: (I) Conception and design: YL Chen, LX Guan, XL Song, X Wang; (II) Administrative support: YL Chen; (III) Provision of study \\ materials or patients: LX Guan, X Wang, X Zhang; (IV) Collection and assembly of data: BL Chen; (V) Data analysis and interpretation: XL Song, \\ LM Liu; (VI) Drafting of manuscript: All authors; (VII) Final manuscript approval: All authors. \\ \#These authors contributed equally to this work. \\ Correspondence to: Yue-Lai Chen, PhD. Yueyang Hospital of Integrated Traditional Chinese and Western Medicine, Shanghai University of \\ Traditional Chinese Medicine, 110 Ganhe Rd, Shanghai 200437, China. Email: chen_yuelai@163.com.
}

\begin{abstract}
Background: Stress urinary incontinence (SUI) is defined as involuntary leakage of urine from the external urethra due to increased abdominal pressure, for example, upon sneezing, coughing, or exercise. Acupuncture is an effective therapy for patients with SUI, although objective evidence of its benefits or mechanism of action is limited. Patients with SUI often harbor structural changes of pelvic floor, the parameters of which are measurable from various perspectives and in multiple dimensions, dynamically and comprehensively, through transperineal ultrasound (TPUS). The status of such changes may then be assessed following acupuncture procedures. In the present investigation, TPUS serves to gauge the immediate effects of acupuncture on pelvic floor structures in female patients with SUI.
\end{abstract}

Methods: This protocol calls for a prospective, randomized, controlled, and single-blinded study of 72 female patients with SUI, each randomly assigned as test or control group members. The test group is subjected to one-time acupuncture at the Zhongii (RN3) acupoint for a period of $10 \mathrm{~min}$, whereas the control group undergoes sham acupuncture in the same manner. In both groups, TPUS imaging of pelvic floor is performed before, during, and immediately after acupuncture procedures. Bladder neck mobility (BND), urethral rotation angle (URA), retrovesical angle (RVA), lowest point of bladder (BN-S), and presence/ absence of urethral funneling or bladder bulging are then recorded as outcome measures.

Discussion: Above efforts are intended to assess real-time pelvic floor structural changes in women undergoing acupuncture for SUI. The subsequent findings may help objectively document the efficacy of acupuncture in this setting and clarify its mechanism of action.

Trial registration: Registration with the Chinese Clinical Trial Registry (ChiCTR200041559) (http:// www.chictr.org.cn/edit.aspx?pid=64591\&htm=4), was effective December 29, 2020.

Dates of study= $12 / 19 / 2020$ to $06 / 30 / 2022$

Keywords: Acupuncture; transperineal pelvic floor ultrasound; stress urinary incontinence (SUI); pelvic floor structure

^ ORCID: Li-Xin Guan, 0000-0002-5308-0599; Xiu-Ling Song, 0000-0002-3280-7189; Yue-Lai Chen, 0000-0001-7246-9420. 
Submitted Mar 23, 2021. Accepted for publication Jun 04, 2021.

doi: 10.21037/apm-21-662

View this article at: https://dx.doi.org/10.21037/apm-21-662

\section{Introduction}

SUI is a common and frequently occurring disorder in adult women. The International Continence Society defines stress urinary incontinence (SUI) as involuntary leakage of urine from the external urethra due to increased abdominal pressure, often upon sneezing, coughing, or exercise (1). Current research shows a median prevalence of $27.6 \%$ for SUI among females globally (2), with an $18.9 \%$ prevalence of SUI recorded for adult women in China. The prevalence of SUI also appears to increase with age, peaking at $28.0 \%$ in the 50- to 59-year age group (3). SUI restricts activities of daily living in women, seriously affecting their quality of life, and imposes a huge psychologic burden (4). Medical treatment of SUI primarily includes lifestyle interventions, pelvic floor muscle training (PFMT), electrical stimulation, drug therapy, and surgery (3). In patients with mild to moderate SUI, lifestyle interventions and PFMT constitute the chief modes of treatment. Such interventions may help to an extent, but due to time requirements, compliance suffers. Drug therapy has some adverse effects (AEs) as well, and electrical stimulations are invasive, potentially causing greater discomfort. Ultimately, surgical treatment of certain patients is appropriate.

According to our preliminary clinical research, acupuncture (as one of various treatments for SUI) (5-8) has a number of advantages, including convenience, quick initiation, significant curative impact, limited AEs, and favorability among patients. However, evidentiary support for its immediate benefits in this setting is still lacking. Patients with SUI often show pelvic floor muscle weakness, fascial and ligamentous laxation, and other functional or structural abnormalities of pelvic floor (9-12). Deficient pelvic floor structural support predisposes to bladder neck and proximal urethral overactivity. Once abdominal pressure increases, bladder pressure exceeds urethral pressure, and urinary incontinence is apt to occur. Pelvic floor structure and SUI are thus closely linked $(13,14)$. Major indicators thereof include bladder neck mobility (BND), urethral rotation angle (URA), retrovesical angle (RVA), lowest point of bladder (BN-S), urethral funneling, and bladder bulging (15).

Pelvic floor ultrasound presently offers accurate and comprehensive assessment of structural changes in pelvic floor from differing perspectives and in multidimensional fashion, enabling early diagnosis of SUI or detection of relevant morphologic alterations that reflect therapeutic efficacy (16). Consequently, the trial detailed herein uses transperineal ultrasound (TPUS) to gauge subsequent real-time pelvic floor structural changes in female patients subjected to acupuncture (sham acupuncture in controls) for SUI. Objective measures of immediate aftereffects are duly generated, building scientific evidence for clinical studies of this nature going forward.

We present the following article in accordance with the SPIRIT reporting checklist (available at https://dx.doi. org/10.21037/apm-21-662).

\section{Methods}

\section{Study design}

A prospective, randomized, controlled, and single-blinded trial was devised to document real-time changes in pelvic floor structure following acupuncture in female patients with SUI, each randomly assigned as a test or control subject. A schematic of study progression is included as Figure 1, and Table 1 lists the schedule of trial enrollment, interventions, and assessments.

This sequentially conducted trial is slated for 72 female participants with SUI, recruited by the Xuhui branch of Zhongshan Hospital at Fudan University through posters, hospital websites, and network clinics. Research assistants are charged with recruitment, relying on a physician for diagnosis of SUI, an acupuncturist for participant treatments, and a sonographer for ultrasound imaging. One-hour urine pad and urine tests are done prior to admission. Once required written consent is granted, participants randomly serve (1:1 ratio) as test or control subjects. In the test group, members submit to acupuncture at the Zhongji (RN3) acupoint for a 10-min period, whereas the control group undergoes sham acupuncture in the same manner. The two groups receive interventions by the same experienced (>2 years) acupuncturist. Ultimately, BND, URA, RVA, and BN-S in resting and Valsalva states, as well as presence/absence of urethral funneling and bladder bulging during Valsalva, are assessed by TPUS of 


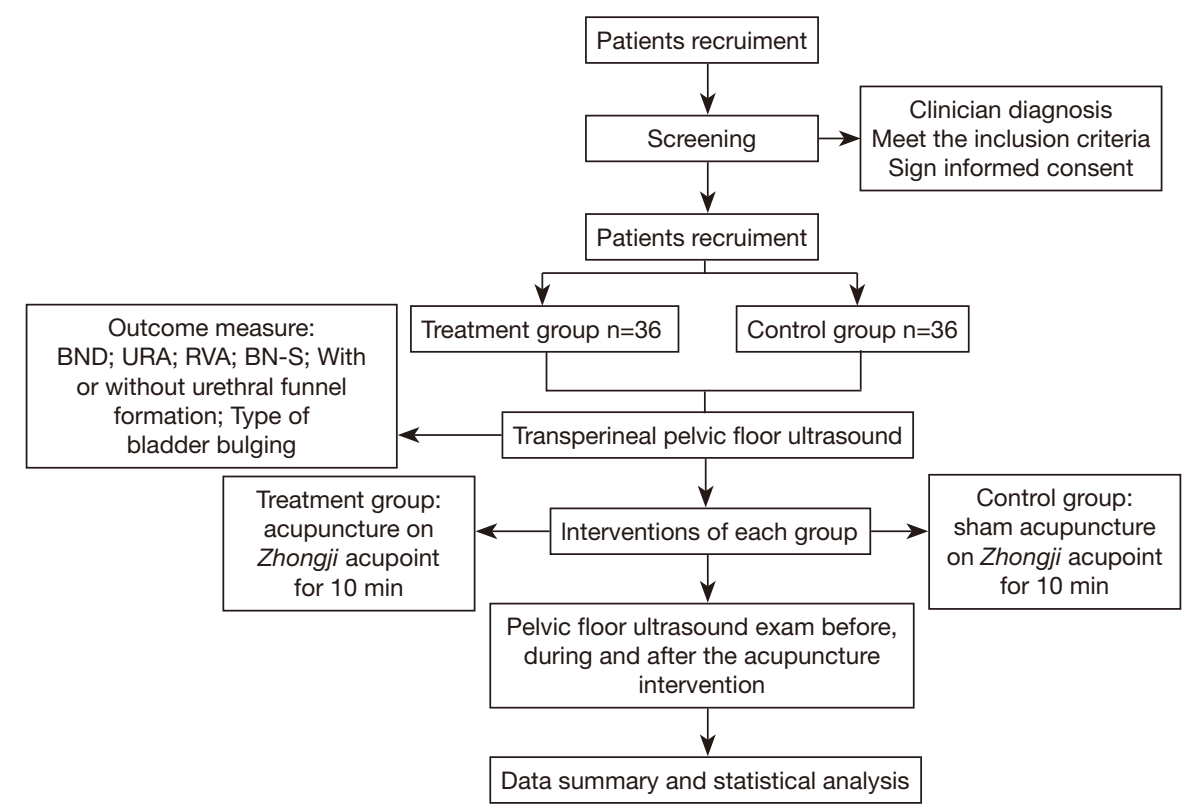

Figure 1 Schematic of study design.

Table 1 Schedule of trial enrollment, interventions, and assessments

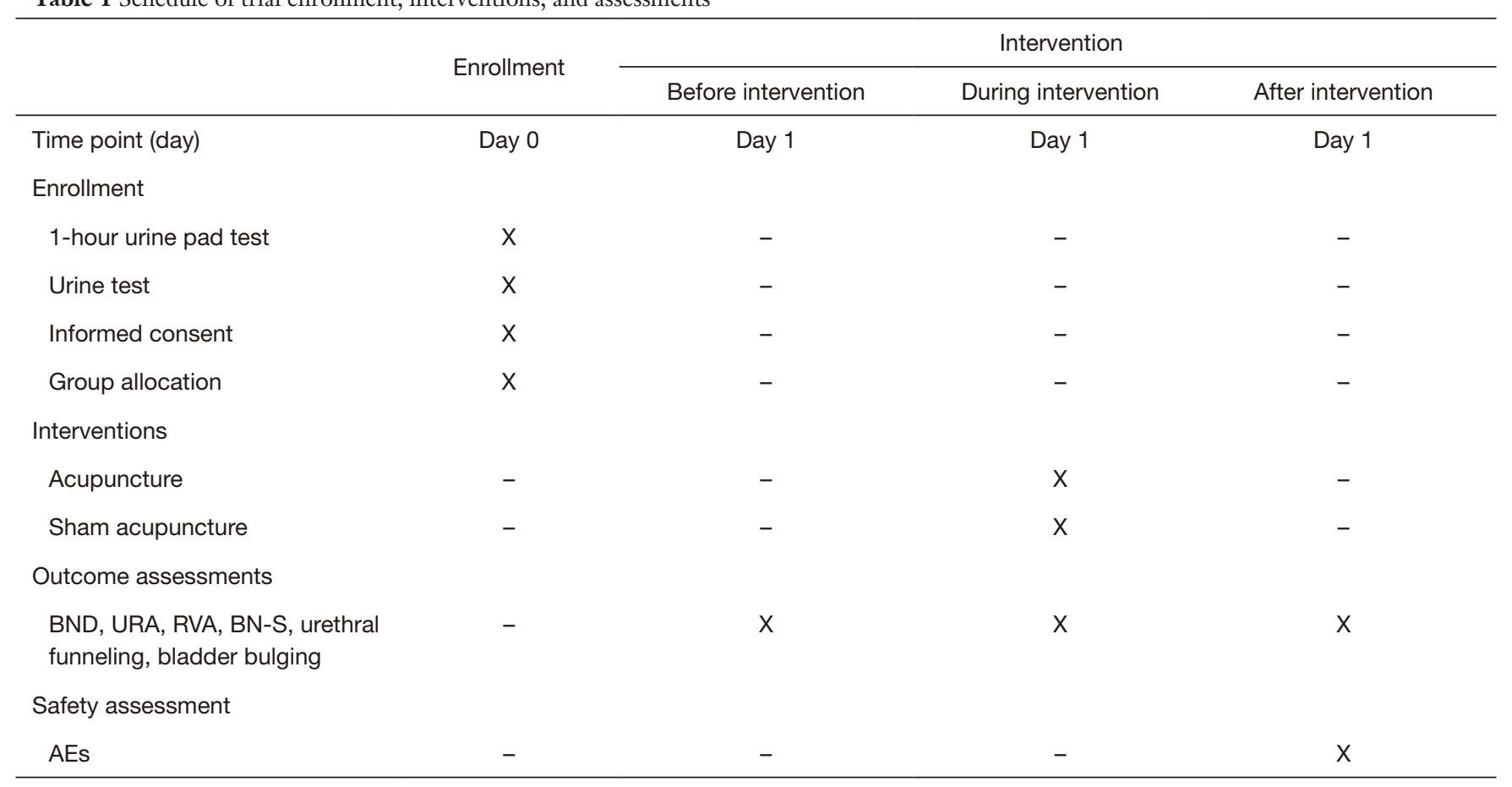

BND, bladder neck mobility; URA, urethral rotation angle; RVA, retrovesical angle; BN-S, lowest point of bladder; AEs, adverse events. 
pelvic floor before, during, and immediately after actual or sham acupuncture procedures. Outcome assessments are performed by researchers uninvolved in treatments and blinded to patient allocation. AEs are recorded after each intervention.

\section{Ethics and consent to participate}

Ethical validity of the study has been assessed and approved by the Chinese Ethics Committee of Registering Clinical Trials (ChiECRCT20200408). This study adheres to Recommendations for Interventional Trials (SPIRIT) (17). All participants must grant informed written once provided with trial details (i.e., study aim, eligibility criteria, course of intervention, and potential benefits/risks) by researchers. All enrollees have the right to withdraw at any time, and use of their personal data is confined to this study only. The trial is formally listed in the Chinese Clinical Trials Registry (registration number ChiCTR2000041559), to which any change in protocol will be directly reported.

\section{Participant characteristics}

\section{Diagnostic criteria}

Diagnosis of SUI in female patients was stipulated as follows, abiding by recommendations of the 6th International Consultation on Incontinence (1): (I) increased abdominal pressure triggered by sneezing, coughing, laughing, exercise, or other events, causing involuntary external urethral urinary leakage; (II) 1-hour urine pad test showing weight gain $>1 \mathrm{~g}$; (III) absence of other urinary symptoms, such as frequency or urgency.

Leakage during the 1-hour urine pad test reflects the degree of SUI as follows: (I) $\leq 1 \mathrm{~g}$ (normal); (II) $>1 \mathrm{~g}$ but $<10 \mathrm{~g}$ (mild); (III) $\geq 10 \mathrm{~g}$ but $<50 \mathrm{~g}$ (moderate); and (IV) $>50 \mathrm{~g}$ (severe).

\section{Inclusion criteria}

The following eligibility criteria were established: (I) clinical signs and symptoms (above) equated with mild to moderate SUI; (II) married women 35-70 years of age; and (III) voluntary study participation, willing to sign informed consent.

\section{Exclusion criteria}

The following criteria were considered grounds for exclusion at study onset: (I) history of surgically treated urinary incontinence or intrapelvic surgery; (II) urologic deformity or organic disease of pelvic cavity; (III) pelvic organ prolapse $\geq$ grade II; (IV) symptomatic urinary tract infection; (V) residual urinary volume $>30 \mathrm{~mL}$; (VI) inability to walk, climb stairs, or run; (VII) inability to cease current bladder regulatory medication (threatening to confound results); (VIII) serious systemic disease (heart, brain, liver, kidney, or blood), mental disorder, diabetes, multiple system atrophy, and cauda equina or medulla spinalis disease; (IX) ongoing pregnancy or lactation; $(\mathrm{X})$ pacemaker placement; (XI) allergy to metal; or (XII) fear of acupuncture.

\section{Dropout criteria}

Participant removal during the course of study in warranted for any of the following reasons: (I) poor compliance or inability to follow trial protocol; (II) failure to execute standard Valsalva maneuvers, impacting observed results; (III) abnormal pelvic cavity or pelvic floor structure (e.g., urethrovaginal fistula) detected by TPUS; (IV) lack of continued cooperation with researcher due to extreme complications or other serious diseases; and $(\mathrm{V})$ incomplete research data.

\section{Randomization and blinding}

Standard software (SPSS v21.0; IBM Corp, Armonk, NY, USA) allows for simple random grouping, the sequence of which is sealed in an opaque envelope. Eligible participants are randomized according to order of hospital visits, assigned to either test or control group (1:1 ratio).

Actual or sham acupuncture is performed without patient knowledge of the particular intervention. Similarly, the sonographer is blinded to patient assignment, and those collecting, recording, and analyzing data are unaware of interventions received to ensure objectivity and reliability of results.

\section{Treatment protocol}

Given our clinical experience, this trial was focused on the Zhongji acupoint, which according to national standards set by the People's Republic of China (18) resides 4 inches below the navel (Figure 2). All participants submit to single 10-min periods of acupuncture at Zhongji acupoint, performing sham acupuncture in the control group. Supine position is maintained throughout, with knees bent, allowing TPUS imaging before, during, and immediately after interventions. Initially, skin that rims the acupoint is sterilized using cotton dipped in $75 \%$ alcohol. A sterile 


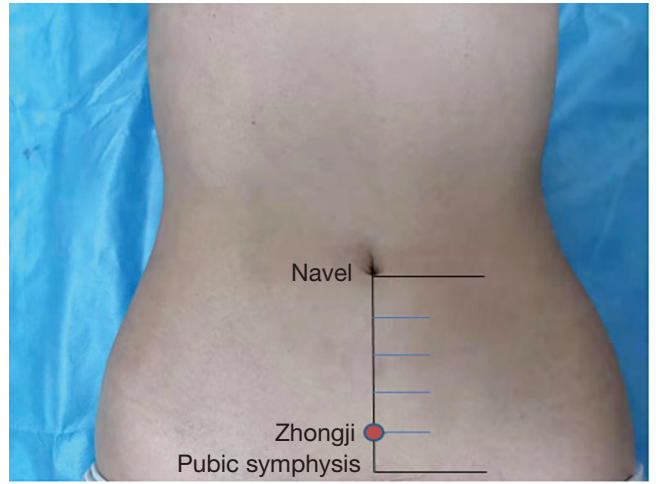

Figure 2 Zhongji acupoint placement: fourth of five evenly dispersed intervals from navel to symphysis pubis.

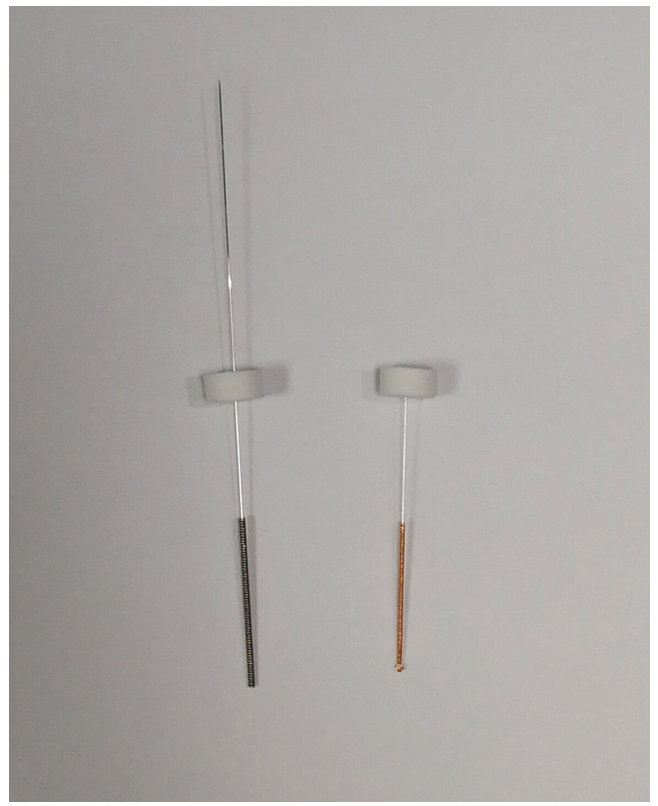

Figure 3 Acupuncture needle (left) and sham needle (right) used in procedures.

adhesive pad (5 mm thick, $10 \mathrm{~mm}$ across), with or without a hole, is then applied at the Zhongji. These pads are composed a special sponge material, with adhesive on one side that affixes to skin (Suzhou Medical Appliance Factory, Suzhou City, China). Pads with central holes are applied to test subjects, those without holes reserved for the control group. The pads serve to mark acupoint sites and ensure blinding beyond the acupuncturist. Both sonographer and acupuncturist will inform every participant of pelvic floor details and reassure them during procedural steps to encourage adherence.

\section{Test group}

TPUS of pelvic floor is also conducted before, during, and immediately after 10 -min periods of acupuncture. The needles used [Hwato brand (0.30-mm gauge, $75 \mathrm{~mm}$ long); Suzhou Medical Appliance Factory] are inserted obliquely into the Zhongji, through the adhesive pad, to a depth of 25-30 $\mathrm{mm}$ (Figure 3). They are then rotated to produce heaviness, numbness, and aching sensations (known as De $Q i)$ of the bladder, perineum, and urethra, remaining in place for $10 \mathrm{~min}$.

\section{Control group}

Control subjects are subjected to 10 -min periods of sham acupuncture (as above), obtaining TPUS studies of pelvic floor before, during, and immediately thereafter. The blunt needle used pierces the pad and touches skin (without breach), remaining in place for a 10 -min period.

\section{Outcome measures}

All TPUS images are acquired using a Voluson S8 system (GE Healthcare, Chicago, IL, USA) equipped with abdominal convex array $(2-5 \mathrm{MHz})$ and intracavity (5-9 MHz) probes (Figure 4). The following parameters are routinely measured: (I) BND, shift in bladder neck relative to lower edge of pubic symphysis (as horizontal reference line) during resting and Valsalva states; (II) URA, difference in urethral tilt angles (UTAs, formed by proximal urethra and bodily longitudinal axis) at rest and during maximum Valsalva if urethra does not cross bodily longitudinal axis during Valsalva (otherwise values are summed); (III) RVA, angle formed by posterior bladder wall (trigonal area) and proximal urethra, determined at rest and during Valsalva; (IV) BN-S, distance from lowest point of bladder to posterior lower edge of pubic symphysis (as horizontal reference line), measured at rest and during Valsalva (value negative if below reference line, positive if above); (V) presence/absence of urethral funneling, indicating whether or not internal urethral orifice is open during Valsalva (i.e., forming a funnel); and (VI) presence/absence of bladder bulging, marked by bladder neck reaching or falling below a reference line during Valsalva.

\section{Safety evaluation}

Any AE, such as broken or retained needles, fainting, unbearable tingling at needle insertion sites, severe postacupuncture pain $>2$ hours, hematoma, infection, abscess, other discomfort after acupuncture (i.e., fatigue, dizziness, 


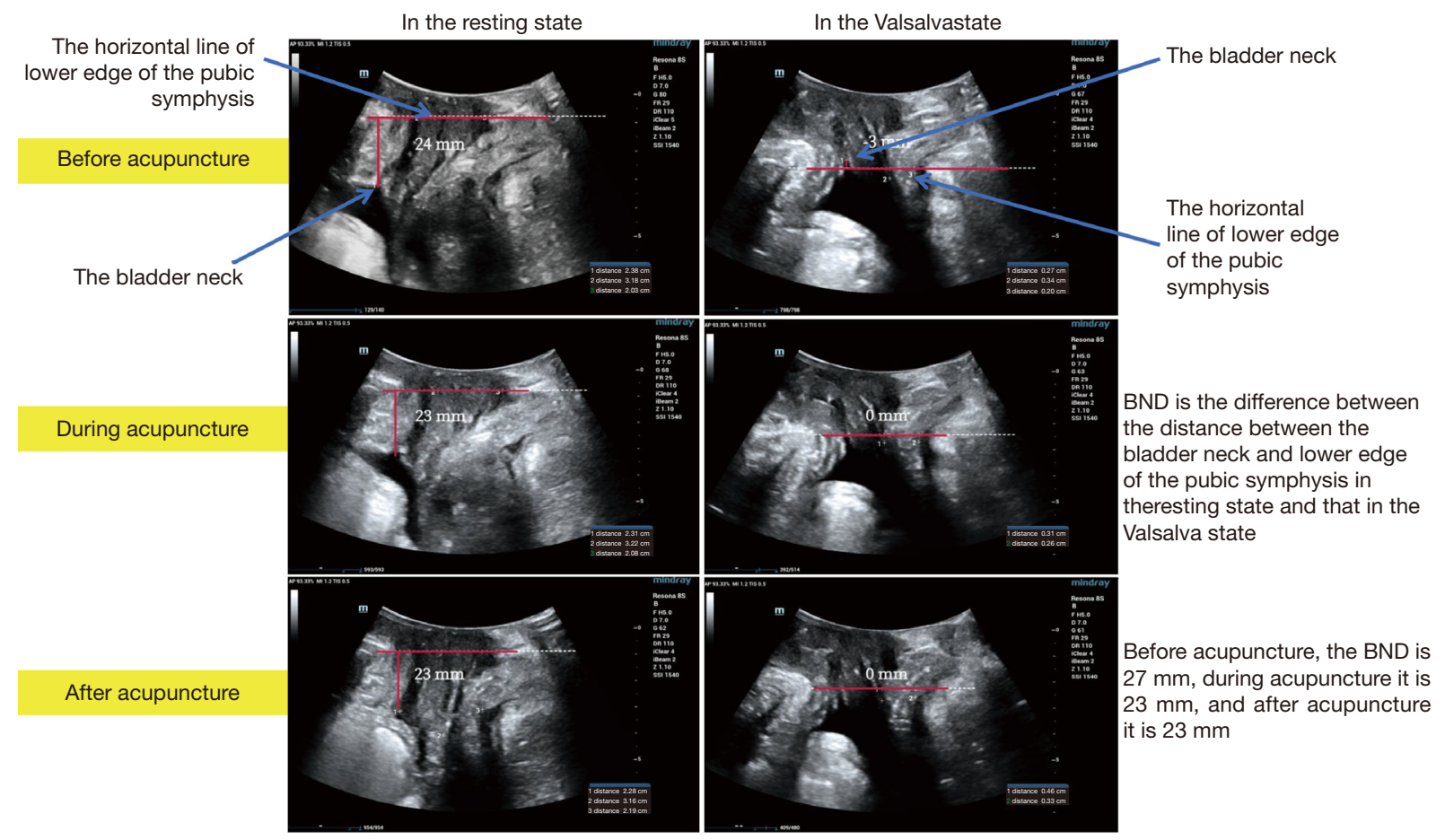

Figure 4 The TPUS images acquired using a Voluson S8 system (GE Healthcare, Chicago, IL, USA) equipped with abdominal convex array $(2-5 \mathrm{MHz})$ and intracavity $(5-9 \mathrm{MHz})$ probes. TPUS, transperineal ultrasound.

palpitations, or headache), and any unforeseen events will be monitored and recorded in a case report form (CRF).

\section{Data collection, management, and monitoring}

In this trial, trained clinical research staff will generate CRFs for all participants, with investigators regularly monitoring or auditing data collection to ensure good quality. Two trained assistants will enter all collected data into a computer for warehousing, enlisting another independent supervisor to check and validate data accuracy and consistency. All hard-copy documents and corresponding electronic data will be stored separately and preserved for at least 5 years after study completion. Any changes to the protocol must be approved by the Ethics Committee. A formal data monitoring committee is not warranted, given the small patient sampling and the low-risk nature of acupuncture.

\section{Statistical analyses}

\section{Sample size}

The sample size for this trial was based on a preliminary study of ours, selecting change in BND as primary outcome measure. As preliminary findings, mean postprocedural change in BND (relative to baseline) was $3.95 \pm$ standard deviation (SD) of 2.86 for the test group, compared with a mean of $1.11 \pm 3.48$ for controls. Sample size was calculated using the following formula (19).

$$
n=\frac{2\left(Z_{\alpha / 2}+Z_{\beta}\right)^{2} \times \sigma^{2}}{\left(\mu_{2}-\mu_{1}\right)^{2}}
$$

In this instance, we set inspection level $(\alpha)$ at 0.05 and test power at 0.9 , so $\beta=1-0.9=0.1, \mu_{1}=3.95, \mu_{2}=1.11$, and $\sigma=3.48$ for two-sided tests. Allowing for $10 \%$ loss during follow-up, at least 36 patients are required for each group, for a total of 72 patients overall.

\section{Data handling}

The platform for data analytics is SPSS (v21.0; IBM Corp), expressing normally distributed and skewed quantitative data as mean \pm SD and median [interquartile range (IQR), P25 - P75], respectively. Student's $t$ test and repeated measures ANOVA will be applied to normally distributed quantitative variables, subjecting skewed quantitative variables to nonparametric testing. Qualitative data will be expressed as numbers and percentages or composition ratios and analyzed 
by chi-square, Fisher's exact, or Wilcoxon rank-sum test, with a two-sided $\mathrm{P}$ value $<0.05$ deemed significant.

\section{Discussion}

Recent clinical studies (5-8) have proven the efficacy of acupuncture in treating SUI, although most have invoked subjective scales, such as the International Consultation on Incontinence Questionnaire-Urinary Incontinence Short Form, for this purpose. Despite the greater objectivity of a urine pad test, it does not align with SUI pathophysiology to yield objective clinical evidence. Currently, there is little published research on the mechanism of acupuncture in the setting of SUI. This trial uses TPUS technology to demonstrate how acupuncture may impact pelvic floor structure in the treatment of SUI. The Zhongji is one acupoint of the Ren meridian and is the $M u$ acupoint of bladder, involving intersection of three $\mathrm{Zu}$-yin meridians with the Ren meridian. It may promote bladder function and aid in urinary restraint, helping to treat urogenital diseases (20). The Zhongji acupoint is emblematic of localized acupuncture effects and is the most direct acupoint for treating disorders of bladder dysfunction (21).

In a previous clinical study (22), sham acupuncture technique has been validated, proving is merit in underscoring the true efficacy of acupuncture procedures. Our participants receive interventions separately to avoid any intercommunication, and researchers are blinded to types of interventions, ensuring objectivity and eliminating bias. If the test group outperforms controls in patients treated for SUI, the immediate effects of acupuncture on pelvic floor structure are thus objectively confirmed.

The diagnostic criteria formulated herein adhered to recommendations of the 6th International Consultation on Incontinence for female patients with SUI, specifically those with mild to moderate degrees of impairment. Cases of overactive bladder, mixed urinary incontinence, or urge incontinence were deliberately excluded, and urodynamic devices were not permitted due to their clinically distinguishable influence.

It is apparent that SUI and abnormal pelvic floor structure are closely linked $(13,14)$. Acupuncture ostensibly helps improve SUI symptoms by mitigating such aberrations. Use of TPUS in patients with SUI serves to characterize the dynamics of pelvic floor structure and provide an objective basis for treatment plans. Its utility is substantial, offering reliability, image reconstruction, and measurement accuracy (23). Use of ultrasound to document real-time pelvic floor structural changes in the context of SUI provides objective imaging evidence of the benefits that acupuncture confers and clarifies its mechanism of action.

\section{Acknowledgments}

We would like to thank BioMed Proofreading LLC (www. biomedproofreading.com) for English language editing.

Funding: This work was supported by grants from the following sources: National Natural Science Foundation of China (No. 81674090); Shanghai Pudong District Famous TCM Experts Training Program (No. PWRzm2020-01); Shanghai Leading Talent Program (No. 2021-013); and Shanghai Municipal Health Commission (No.201940011); Yueyang Hospital of Integrated Traditional Chinese and Western Medicine and Shanghai University of Traditional Chinese Medicine provided professional guidance.

\section{Footnote}

Reporting Checklist: The authors have completed the SPIRIT reporting checklist. Available at https://dx.doi. org/10.21037/apm-21-662

Peer Review File: Available at https://dx.doi.org/10.21037/ apm-21-662

Conflicts of Interest: All authors have completed the ICMJE uniform disclosure form (available at https://dx.doi. org/10.21037/apm-21-662). YLC reports that this work was supported by Grants from The National Natural Science Foundation of China (No. 81674090); Shanghai Pudong district famous TCM experts training program (No. PWRzm2020-01); Shanghai Leading Talent Program (No. 2021-013); Shanghai Municipal Health Commission (No.201940011). The other authors have no conflicts of interest to declare.

Ethical Statement: All participants must grant written informed consent once trial details are fully presented. The authors are accountable for all aspects of work, ensuring that questions related to accuracy or integrity of any part are appropriately investigated and resolved. Ethical validity of the study has been assessed and approved by the Chinese Ethics Committee of Registering Clinical Trials (ChiECRCT20200408).

Open Access Statement: This is an Open Access article 
distributed in accordance with the Creative Commons Attribution-NonCommercial-NoDerivs 4.0 International License (CC BY-NC-ND 4.0), which permits the noncommercial replication and distribution of the article with the strict proviso that no changes or edits are made and the original work is properly cited (including links to both the formal publication through the relevant DOI and the license). See: https://creativecommons.org/licenses/by-nc-nd/4.0/.

\section{References}

1. Abrams P, Cardozo L, Wagg A, et al. Sixth International Consultation on Incontinence. Bristol, UK: International Continence Society, 2016:2551-6.

2. Minassian VA, Drutz HP, Al-Badr A, et al. Urinary incontinence as a worldwide problem. Int J Gynaecol Obstet 2003;82:327-38.

3. Zhu L, Sun Z. Guidelines for the diagnosis and treatment of stress urinary incontinence in women (2017). Chinese Journal of Obstetrics and Gynecology 2017;52:289-93.

4. Li ZY, Zhu L. Epidemiological status of female stress urinary incontinence. Journal of Practical Obstetrics and Gynecology 2018;34:161-2.

5. Liu Z, Liu Y, Xu H, et al. Effect of Electroacupuncture on Urinary Leakage Among Women With Stress Urinary Incontinence: A Randomized Clinical Trial. JAMA 2017;317:2493-501.

6. Tang KM, Shen R, Jiang F, et al. Therapeutic Evaluation of Acupuncture for Female Stress Urinary Incontinence. Shanghai Journal of Acupuncture and Moxibustion 2016;35:1439-41.

7. Hou WG, Ming SR, Tang KM, et al. Preventive and Therapeutic Effects of Electroacupuncture in Treating Mild-moderate Female Stress Urinary Incontinence. Shanghai Journal of Acupuncture and Moxibustion 2017;36:956-9.

8. Zheng HM, Xu SF, Yin P, et al. Observation on Shortterm and Long-term Therapeutic Effects of Electroacupuncture on Mild and Moderate Stress Urinary Incontinence. World Journal of Integrated Traditional and Western Medicine 2015;10:191-3, 209.

9. Li XW, Wang JL. Causes and pathogenesis of urinary incontinence. Journal of Practical Obstetrics and Gynecology 2018;34:162-4.

10. Wood LN, Anger JT, et al. Urinary incontinence in women. BMJ 2014;349:g4531.

11. Markland AD, Richter HE, Fwu CW, et al. Prevalence and trends of urinary incontinence in adults in the United
States, 2001 to 2008. J Urol 2011;186:589-93.

12. DeLancey JO. Structural support of the urethra as it relates to stress urinary incontinence: the hammock hypothesis. Am J Obstet Gynecol 1994;170:1713-20; discussion 1720-3.

13. Luo X. New concept of female pelvic floor anatomy. Chinese Journal of Practical Gynecology and Obstetrics 2006;22:78-80.

14. Ashton-Miller JA, Howard D, DeLancey JO. The Functional Anatomy of the Female Pelvic Floor and Stress Continence Control System. Scand J Urol Nephrol Suppl 2001;(207):1-7; discussion 106-25.

15. Li K, Xie W, Guo Z, et al. Application of pelvic floor ultrasonography in measuring angular parameters in women with stress urinary incontinence. Chinese Journal of Medical Imaging 2017;25:937-40.

16. Zhang XL, Huang ZP, Mao YJ, et.al. Practical ultrasonic diagnosis of pelvic floor. Beijing: People's Medical Publishing House 2018:5.

17. Chan AW, Tetzlaff JM, Gøtzsche PC, et al. SPIRIT 2013 explanation and elaboration: guidance for protocols of clinical trials. BMJ 2013;346:e7586.

18. Huang LX. National Standard of the People's Republic of China. Acupoints. Beijing: Standards Press of China 2006:13-34.

19. Glueck DH. Sample Size Calculations in Clinical Research 2nd edition by CHOW, S.-C., SHAO, J., and WANG, H. Biometrics 2008;64:1307-8.

20. Cheng $\mathrm{XN}$. Chinese acupuncture and moxibustion. Beijing: Foreign language press, 1990:225.

21. Zhao J. Discussion on "nearby action", the basic function of acupoints. Zhongguo Zhen Jiu 2015;35:1196-8.

22. Huang H, Song X, Zhao L, et al. Opposing needling for analgesia and rehabilitation after unilateral total knee arthroplasty: a randomized, sham-controlled trial protocol. Trials 2020;21:385.

23. Chen MZ, Zhan JX. Value of transperineal fourdimensional ultrasound in diagnosis of female stress urinary incontinence. Journal of Clinical Ultrasound in Medicine 2017;19:344-6.

Cite this article as: Guan $\mathrm{LX}$, Song $\mathrm{XL}$, Wang $\mathrm{X}$, Zhang $\mathrm{X}$, Liu LM, Chen BL, Chen YL. Immediate effects of Zhongji point acupuncture on pelvic floor structure in female patients with stress urinary incontinence: a randomized, single-blind, and sham-controlled clinical trial protocol. Ann Palliat Med 2021;10(7):8292-8299. doi: 10.21037/apm-21-662 